\title{
Emergence of helicity in rotating stratified turbulence
}

\author{
Raffaele Marino, ${ }^{1}$ Pablo D. Mininni, ${ }^{1,2}$ Duane Rosenberg, ${ }^{3}$ and Annick Pouquet ${ }^{1,4}$ \\ ${ }^{1}$ National Center for Atmospheric Research, P.O. Box 3000, Boulder, Colorado 80307, USA \\ ${ }^{2}$ Departamento de Física, Facultad de Ciencias Exactas y Naturales, Universidad de Buenos Aires and IFIBA, CONICET, \\ Ciudad Universitaria, 1428 Buenos Aires, Argentina \\ ${ }^{3}$ National Center for Computational Sciences, Oak Ridge National Laboratory, P.O. Box 2008, Oak Ridge, Tennessee 37831, USA \\ ${ }^{4}$ Department of Applied Mathematics, University of Colorado at Boulder, Boulder, Colorado 80309, USA
}

(Received 8 June 2012; revised manuscript received 25 January 2013; published 25 March 2013)

\begin{abstract}
We perform numerical simulations of decaying rotating stratified turbulence and show, in the Boussinesq framework, that helicity (velocity-vorticity correlation), as observed in supercell storms and hurricanes, is spontaneously created due to an interplay between buoyancy and rotation common to large-scale atmospheric and oceanic flows. Helicity emerges from the joint action of eddies and of inertia-gravity waves (with inertia and gravity with respective associated frequencies $f$ and $N$ ), and it occurs when the waves are sufficiently strong. For $N / f<3$ the amount of helicity produced is correctly predicted by a quasilinear balance equation. Outside this regime, and up to the highest Reynolds number obtained in this study, namely $\operatorname{Re} \approx 10000$, helicity production is found to be persistent for $N / f$ as large as $\approx 17$, and for $\mathrm{ReFr}^{2}$ and ReRo ${ }^{2}$, respectively, as large as $\approx 100$ and $\approx 24000$.
\end{abstract}

DOI: 10.1103/PhysRevE.87.033016

PACS number(s): 47.32.Ef, 47.27.-i, 47.55.Hd

\section{INTRODUCTION}

Symmetry breaking is a fundamental concept which has been quite fruitful in many physical applications [1]. For a fluid the simplest way to break symmetry is to introduce helicity. In that case, the velocity covariance tensor is still expressible in terms of the magnitude of the distance between points, i.e., the fluid still has isotropic statistics, but mirror symmetry is broken whereby the covariance matrix has an antisymmetric component which can be shown to be proportional to the total helicity $H_{V}=\langle\mathbf{u} \cdot \boldsymbol{\omega}\rangle$, which is defined as the correlation between the velocity $\mathbf{u}$ and its curl, the vorticity $\boldsymbol{\omega}$. Helical structures abound in nature, from macroscopic organisms to elastomers; helical structures can cause erosion in river bends [2], and alter nutrient mixing properties in estuaries, in particular when interacting with tidal flows [3]. Helical flows are observed as well in the atmosphere, in dust devils, tornadoes, and hurricanes [4].

Helicity is an invariant of motion of the nondissipative fluid equations involving the topology of field lines [5], including in the presence of rotation, but not with stratification, where instead the potential vorticity is invariant and is essential in determining structures such as sharp jets in planetary atmospheres [6]. Invariants are known to play a fundamental role in turbulence since the nonlinear terms have to preserve such invariants at the level of triadic interactions in the incompressible case. However, helical (corkscrew) motions do not seem to alter the dynamics of homogeneous isotropic turbulence in the absence of both rotation and stratification, with the kinetic energy and helicity spectral densities [respectively, $E_{V}(k)$ and $H_{V}(k)$, with $\int E_{V}(k) d k=\frac{1}{2}\left\langle u^{2}\right\rangle$ and $\int H_{V}(k) d k=H_{V}$ ] both following a Kolmogorov spectrum. This implies a slow $\sim 1 / k$ decay of the relative helicity in Fourier space

$$
\hat{\Sigma}(k)=\frac{H_{V}(k)}{k E_{V}(k)},
$$

with $\sigma_{V}(\mathbf{x})=\cos (\mathbf{u}, \boldsymbol{\omega})$ the degree of alignment between velocity and vorticity in configuration space. However, it is straightforward to show that helicity is created point-wise by the alignment of vorticity and pressure or shear gradients [7], and it is observed to be strong $\left(\sigma_{V} \approx \pm 1\right)$ in the vortex filaments that are ubiquitous in isotropic fluid turbulence at a small scale.

Invariants are also the stepping stone to determine inertial range behavior in turbulent flows; this principle is at the basis of statistical mechanics that has proven useful in predicting, for example, the inverse cascade of energy for a two-dimensional fluid [8]. In fact, a recent direct numerical simulation of the ideal three-dimensional fluid equations in the absence of waves showed that, at intermediate times, a Kolmogorov spectrum develops at large scale, the effective dissipation for the large-scale fluid being produced by the eddy viscosity emanating from the small-scale equilibrated modes [9]. However, nonconserved quantities can also play an important role through other mechanisms such as interactions with waves and large-scale hydrostatic and geostrophic balance [10].

When the fluid is conducting, magnetic helicity is an invariant in the ideal case and is central to minimum energy equilibria in plasmas such as in spheromaks, or in solar coronal mass ejections [11]. It is also known that the generation of large-scale magnetic fields occurs due to small-scale mechanic helicity $H_{V}$, and that in the presence of both rotation and stratification, helicity is created and thus a dynamo is facilitated in a wide variety of astrophysical settings [12]. In the context of this work, it is important to note that although in this case the mechanic helicity is not an invariant any longer, it still plays an essential role in determining the scaling of the fields at large scales.

Rotating stratified turbulence is important in the atmosphere and oceans, playing a crucial role in their dynamics. In the presence of waves, advective nonlinear interactions responsible for the complexity of turbulent flows have to compete with the waves and an equilibrium can be reached at some scale and 
broken at others, the best known example perhaps being the difference between the Garret-Munk and the Phillips spectra in internal waves in lakes or the oceans $[13,14]$, where wave coupling in resonant triads leads to mixing (like in coastal currents [15]), to vertical dispersion [16], and to enhanced dissipation [17]. A particular set of modes plays a major role in the so-called slow manifold for which the frequency of the waves tends to zero, and only turbulent interactions and standing potential vorticity modes remain. When rotation (only) is present in the fluid, strong relative helicity can alter the scaling of the distribution of energy among scales and lead to the occurrence of helical long-lived structures [18].

What happens when stratification is also included? In the remainder of this paper, we address the question of rotating stratified turbulence in the absence of forcing (as studied for example in $[19,20]$ ), but concentrating on the creation of helicity and on the link between the evolution of helicity and the balance of forces such as rotation, stratification, inertia, and pressure gradients, at scales large enough that the effect of nonlinearities (inertia) is small. In the limit of zero nonlinearities, the resulting geostrophic balance is crucial for weather forecasting and simulations of climate change. However, the consequences of the interplay between rotation and stratification, as far as helical motions are concerned, have been mostly ignored except for the pioneering work of Hide [21]. In spite of this, helicity was hypothesized to be important in the atmosphere in the dynamics and persistence of rotating convective storms [22], on the basis of the weakening of nonlinear interactions in the so-called Lamb vector $\mathbf{u} \times \boldsymbol{\omega}$.

It is also interesting that helicity is measured in the context of forecasting storms and tropical tornadoes, in particular in the presence of strong shear, and it can be used as an indicator of storm occurrence [23]. Note that it has been shown that in some cases (using a specific fully helical Beltrami forcing function) that shear is created at large scale in a rotating flow [24].

Since helicity in rotating and stratified flows is no longer an invariant even in the absence of dissipation, its presence in these atmospheric storms can be accounted for, but the physical mechanisms governing its creation, and the structures associated with it, remain unclear. In this paper, we perform a parametric study using direct numerical simulations in which we vary both rotation and stratification. In that framework, we show that a strongly rotating stratified flow can spontaneously create helicity at large scales.

\section{EQUATIONS AND NUMERICAL PROCEDURE}

\section{A. Boussinesq equations and parameters}

We integrate the incompressible Boussinesq equations in the rotating frame, with constant (solid body) rotation $\Omega$ and gravity $g$, anti-aligned in the vertical $(z)$ direction, with $\theta$ the buoyancy (in units of velocity), $w$ the vertical velocity, $P$ the pressure, $v$ the viscosity, and $\kappa$ the diffusivity

$$
\begin{gathered}
\partial_{t} \mathbf{u}+\mathbf{u} \cdot \nabla \mathbf{u}-v \Delta \mathbf{u}=-\nabla P-N \theta \boldsymbol{e}_{z}-2 \Omega \boldsymbol{e}_{z} \times \mathbf{u}, \\
\partial_{t} \theta+\mathbf{u} \cdot \nabla \theta-\kappa \Delta \theta=N w, \\
\nabla \cdot \mathbf{u}=0 .
\end{gathered}
$$

We write $\mathbf{u}=(u, v, w)$ and we take a unit Prandtl number $v=$ $\kappa$. The Brunt-Väisälä frequency is $N=\left[-g \partial_{z} \bar{\theta} / \theta\right]^{1 / 2}$ where $\bar{\theta}$ is the background imposed stratification. In the general case, one has inertia-gravity waves of frequency

$$
\omega_{I G}=k^{-1} \sqrt{N^{2} k_{\perp}^{2}+f^{2} k_{z}^{2}}
$$

with $f=2 \Omega$ (see, e.g., [19,25]).

The Froude, Rossby, and Reynolds numbers are defined, respectively, as

$$
\mathrm{Fr}=\frac{u_{0}}{N L_{0}}, \quad \operatorname{Ro}=\frac{u_{0}}{f L_{0}}, \quad \operatorname{Re}=\frac{u_{0} L_{0}}{v},
$$

with $u_{0}=1$ and $L_{0}=2 \pi / k_{0}$, respectively, the r.m.s. velocity and the scale of the initial conditions. These parameters vary in the range $0.0063 \leqslant \mathrm{Fr} \leqslant 0.2,0.0063 \leqslant \mathrm{Ro} \leqslant 3.24$, and $\mathrm{Re} \approx 4000$ for grids of $256^{3}$ points, while $\mathrm{Re} \approx 10000$ using $512^{3}$ points. Decay is left to occur for 3.6 to 7.2 turn-over times $\tau_{N L}=L_{0} / u_{0}$. The initial velocity field is random, with all three components nonzero, and it is centered around wave numbers $k_{0}=[1,2]$. At $t=0, \theta=0$, and $H_{V} \approx+0.2$. Other initial values have been used as well to ascertain that the results are insensitive to them. Note that we do not attempt to take initially a balanced flow; for the time-stepping point of view there is no need to do so since the resolutions we employ are high enough that the relatively small Froude and Rossby numbers we simulate can be handled with an explicit time stepping resolving the smallest eddy-turn-over time and the smallest Brunt-Väisälä, inertial, and inertia-gravity frequencies. Furthermore, the generation of gravity waves that compete with turbulent eddies is part of the overall dynamics of such flows as the Reynolds number increases.

In the ideal $(v=0)$ case, potential vorticity

$$
q=-f N+f \partial_{z} \theta-N \omega_{z}+\omega \cdot \nabla \theta
$$

is a point-wise invariant, and the total (kinetic plus potential) energy $E_{T}=E_{V}+E_{P}$ is conserved as well (with $E_{P}=\left\langle\theta^{2}\right\rangle / 2$ ), with the respective enstrophies (proportional to dissipation when $v \neq 0$ and $\kappa \neq 0$ )

$$
Z_{V}=\left\langle\omega^{2}\right\rangle, \quad Z_{P}=\left\langle|\nabla \theta|^{2}\right\rangle .
$$

Note that $q$ is quadratic and thus its $L_{2}$ norm is not conserved in general by the truncation of Fourier space used in any spectral method; however, the nonlinear term $\omega \cdot \nabla \theta$ can be neglected in the presence of strong rotation and stratification [26], resulting in a quantity whose $L_{2}$ norm is conserved after truncation of Fourier space.

\section{B. GHOST code and the runs}

The numerical simulations have been carried out using the Geophysical High-Order Suite for Turbulence (GHOST) code. GHOST is a pseudospectral framework that hosts a variety of partial differential equation (PDE) solvers optimized for studying turbulence in a $[0,2 \pi]^{3}$ triperiodic box, and with second- or fourth-order explicit Runge-Kutta time stepping schemes. Using a cubic box and an explicit time stepping method allows in principle, given the parameters are right, for resolving all scales including the Ozmidov scale and beyond, when isotropy recovers (see, e.g., [27] for the purely rotating case). A classical 2/3 de-aliasing rule is used, meaning 
TABLE I. List of runs analyzed in this paper with some characteristic parameters: Run number $n_{R}$, linear resolution $n_{p}$, Reynolds Re, Froude Fr, and Rossby Ro numbers. A star in the "in" column indicates points that are in the scatter plot with $N / f<3$, and two stars indicate those in the plot with $\mathrm{ReFr}^{2}<20$ or $\mathrm{ReRo}^{2}<20$ (see Figs. 4 and 5).

\begin{tabular}{|c|c|c|c|}
\hline$n_{R}$ & $\begin{array}{c}\begin{array}{c}\text { Fr } \\
n_{p}=256^{3}\end{array}\end{array}$ & $\begin{array}{c}\text { Ro } \\
;\end{array}$ & $\begin{array}{c}\text { in } \\
\operatorname{Re}=4189\end{array}$ \\
\hline 1 & 0.0063 & 0.0063 & $*, * *$ \\
\hline 2 & 0.0063 & 0.0127 & $*, * *$ \\
\hline 3 & 0.0063 & 0.0190 & - \\
\hline 4 & 0.0063 & 0.0507 & - \\
\hline 5 & 0.0063 & 0.0728 & $* *$ \\
\hline 6 & 0.0063 & 0.1013 & $*, * *$ \\
\hline 7 & 0.0084 & 0.0084 & $*, * *$ \\
\hline 8 & 0.0127 & 0.0063 & $*, * *$ \\
\hline 9 & 0.0127 & 0.0127 & $*, * *$ \\
\hline 10 & 0.0127 & 0.0190 & $*, * *$ \\
\hline 11 & 0.0127 & 0.0253 & $*, * *$ \\
\hline 12 & 0.0127 & 0.0317 & $* *$ \\
\hline 13 & 0.0127 & 0.0384 & $* *$ \\
\hline 14 & 0.0127 & 0.0507 & - \\
\hline 15 & 0.0127 & 0.1013 & - \\
\hline 16 & 0.0127 & 0.1458 & - \\
\hline 17 & 0.0127 & 0.2111 & $*, * *$ \\
\hline 18 & 0.0253 & 0.0253 & - \\
\hline 19 & 0.0253 & 0.0507 & - \\
\hline 20 & 0.0253 & 0.1013 & - \\
\hline 21 & 0.0253 & 0.2026 & - \\
\hline 22 & 0.0253 & 0.2913 & $*, * *$ \\
\hline 23 & 0.0253 & 0.4054 & - \\
\hline 24 & 0.0507 & 0.0507 & - \\
\hline 25 & 0.0507 & 0.1267 & - \\
\hline 26 & 0.1013 & 0.4224 & - \\
\hline 27 & 0.1013 & 0.8444 & $*, * *$ \\
\hline 28 & 0.1013 & 1.1515 & $*$ \\
\hline 29 & 0.1013 & 1.6888 & $*$ \\
\hline 30 & 0.1266 & 0.1266 & - \\
\hline 31 & 0.2026 & 0.2026 & - \\
\hline 32 & 0.2026 & 0.6079 & - \\
\hline 33 & 0.2026 & 0.8106 & - \\
\hline 34 & 0.2026 & 1.6888 & - \\
\hline 35 & 0.2026 & 2.3268 & $*, * *$ \\
\hline \multirow[t]{2}{*}{36} & 0.2026 & 3.2428 & - \\
\hline & $n_{p}=512^{3}$ & ; & $\mathrm{Re}=10649$ \\
\hline 37 & 0.0063 & 0.0127 & - \\
\hline 38 & 0.0063 & 0.0190 & $*$ \\
\hline 39 & 0.0127 & 0.0190 & $*, * *$ \\
\hline 40 & 0.0127 & 0.0317 & $*, * *$ \\
\hline 41 & 0.0127 & 0.0443 & $* *$ \\
\hline 42 & 0.0127 & 0.0633 & $*, * *$ \\
\hline 43 & 0.0127 & 0.1013 & - \\
\hline 44 & 0.0253 & 0.0507 & $*, * *$ \\
\hline 45 & 0.1013 & 0.4053 & $*$ \\
\hline
\end{tabular}

that for a given resolution of $n_{p}$ points per dimension, the maximum available wave number is $k_{\max }=n_{p} / 3$. The code uses a hybrid MPI/OpenMP parallelization scheme [28] (MPI is the Message Passing Interface library, and OpenMP stands for Open Multiprocessing, an interface to program shared memory environments). The code also has a third level of parallelization with the recent addition of support for graphic processing units (GPUs) and accelerators for the fast Fourier transforms (FFTs). Note that the MPI communication required to complete the multidimensional Fourier transforms is all-to-all. The code uses a "slab" (one-dimensional) domain decomposition among MPI tasks, and OpenMP provides a second level of parallelization within each slab or MPI task. The code can compute in double or single precision based on resolution. GHOST performance has been tested on a variety of platforms, and has been shown to scale linearly up to 98304 processors, with grids up to $6144^{3}$ points. Data are stored at regular intervals and postprocessed, both for quantitative analysis and visualization, the latter being performed using the VAPOR visualization software [29].

In Table I we give the major parameters of the simulations used in this paper. Note that we have restricted our analysis to moderate values of $N / f$, in particular we have for all cases $N / f \geqslant 1 / 2$. This is because, in the purely rotating case $(N \rightarrow 0)$, helicity is exactly conserved and thus as one goes into that parameter regime, the creation of helicity has to become negligible with decreasing $N$ at fixed $f$; furthermore, many geophysical flows are dominated by gravity waves except at the largest scales. There are studies that show, for example, that, for purely rotating flows, a turbulence regime affected by waves develops for $\mathrm{Ro}<0.2$, whereas at intermediate Rossby numbers nonlinear transfer is reduced but the inverse cascade characteristic of the bi-dimensionalization of the flow does not take place [30]. Also, for strong waves (strong rotation or stratification), turbulence barely develops resulting in steep spectra; this is related to the value achieved by the so-called buoyancy Reynolds number $\operatorname{ReFr}^{2}$ defined below, and the equivalent concept for rotating flows, $\mathrm{ReRo}^{2}$. Considering this region of interest in parameter space, and given the constraints of computing in three dimensions without resorting to modeling of the small scales, only a limited exploration of the parameters is performed.

\section{RESULTS}

\section{A. Generation of helicity for small nonlinearity}

As mentioned in the Introduction, helicity is not conserved in a rotating and stratified flow, and thus helicity can in principle be created by the flow evolution. In this section we briefly show how a balance of the forces at large scales can result in net helicity of a preferred sign in the flow. We start from the primitive Boussinesq equations given above and simplify them using several hypotheses. Assuming stationarity, weak nonlinearities, and small dissipation at large scales, it results that the equilibrium level of helicity in rotating stratified turbulence is proportional to $N / f$ and to the correlation between buoyancy and vertical shear. A result consistent with this behavior was originally obtained by Hide [21].

We start with the momentum equation, Eq. (2). As later we will compute time averages, we will assume the system is in a steady state and neglect the time derivative. We will also consider that the viscous effects are small, and neglect 
the dissipative term. These are the two main assumptions we are making at this stage. Without any further assumption, we now compute the vertical derivative of the remaining terms and take the dot product of the result with the velocity $\mathbf{u}$. We therefore obtain the following relation:

$$
\mathbf{u} \cdot \partial_{z}(\mathbf{u} \cdot \nabla \mathbf{u})=-\mathbf{u} \cdot \nabla \partial_{z} P-N w \partial_{z} \theta-2 \mathbf{u} \cdot \partial_{z}\left(\Omega \boldsymbol{e}_{z} \times \mathbf{u}\right),
$$

where the velocity field $\mathbf{u}$ was written with Cartesian components $(u, v, w)$.

The last term in this equation is

$$
2 \mathbf{u} \cdot \partial_{z}\left(\Omega \boldsymbol{e}_{z} \times \mathbf{u}\right)=-f\left(u \partial_{z} v-v \partial_{z} u\right)=f H_{\perp},
$$

where $H_{\perp}$ is part of the total helicity density. Indeed, one can decompose the helicity as $H_{V} \equiv\left\langle H_{\perp}\right\rangle+\left\langle H_{+}\right\rangle$, where the brackets denote an average, and where $H_{\perp}$ is the helicity density associated with $\mathbf{u}_{\perp}$, namely

$$
H_{\perp} \equiv \boldsymbol{u}_{\perp} \cdot\left(\nabla \times \boldsymbol{u}_{\perp}\right),
$$

and $H_{+}$is the remainder, $H_{+}=u \partial_{y} w-v \partial_{x} w+w \omega_{z}$. With strong rotation and stratification, $H_{\perp} \gg H_{+}$, and $H_{\perp}$ alone essentially determines the total helicity. For example, measurements of $\left\langle H_{\perp}\right\rangle_{\perp, z}$ (where the subindices $\perp$ and $z$ indicates the averages are volume averages performed in the horizontal and vertical directions) found in modeling simulations of hurricanes are seen to be two orders of magnitude larger than $\left\langle H_{+}\right\rangle$[31]. Note also that the $H_{\perp}$ density is proportional to the so-called (cell-relative) environmental helicity, when integrated over the vertical (see, e.g., [23]). Using these observations, we now concentrate on $H_{\perp}$, which allows us to derive a simpler expression for helicity production. Thus, we can write in general from Eqs. (4) and (5) that

$$
H_{\perp}=-\frac{1}{f}\left[N w \partial_{z} \theta+\mathbf{u} \cdot \nabla \partial_{z} P+\mathbf{u} \cdot \partial_{z}(\mathbf{u} \cdot \nabla \mathbf{u})\right] .
$$

Our next step is to integrate over volume, in which case the second term vanishes for an incompressible flow, so that after integration we obtain the general expression

$$
\left\langle H_{\perp}\right\rangle_{\perp, z}=-\frac{N}{f}\left\langle w \frac{\partial \theta}{\partial z}\right\rangle_{\perp, z}-\frac{1}{f}\left\langle\mathbf{u} \cdot \partial_{z}(\mathbf{u} \cdot \nabla \mathbf{u})\right\rangle_{\perp, z} .
$$

Equation (8) holds under the assumptions of stationarity, incompressibility, and negligible dissipation. The second term is cubic in the velocity and in a turbulent flow proportional to $\epsilon\left(f L_{z}\right)^{-1}$, where $\epsilon$ is the energy flux, and $L_{z}$ a characteristic vertical scale. For flows with strong rotation and stratification, this quantity is expected to be small. In fact, we verified explicitly that in a certain range of parameters (defined in detail in Sec. III B), the amplitude of the second term in our simulations is smaller than $10-20 \%$ of the first term. As a result, after neglecting this nonlinear term, we finally obtain

$$
\left\langle H_{\perp}\right\rangle_{\perp, z}=-\frac{N}{f}\left\langle w \frac{\partial \theta}{\partial z}\right\rangle_{\perp, z} .
$$

This expression was derived before by Hide [21], in a slightly different form after integrating by parts and assuming periodic boundary conditions, leading to the equivalent expression

$$
\left\langle H_{\perp}\right\rangle_{\perp, z}=\frac{N}{f}\left\langle\theta \frac{\partial w}{\partial z}\right\rangle_{\perp, z} .
$$

It should be noted that the original derivation in [21] assumes the nonlinear term is zero and that the flow is in geostrophic balance. In that case, from hydrostatic balance $\partial_{z} w=0$ and helicity in the flow vanishes. Small nonlinearities are crucial to ensure that the second-order correlator in Eqs. (9) or (10) is nonzero.

We thus conclude that, if nonlinearities are small, the production of helicity in strongly rotating stratified turbulence is such that its equilibrium level is directly proportional to $N / f$, and results from a crucial interplay between rotation and stratification. In the limit of $f \rightarrow \infty$ (no stratification), helicity is exactly conserved. In the limit of $N \rightarrow \infty$, stratification dominates and the evolution of helicity can only be governed by the nonlinear terms, the buoyancy, and the dissipation [32]. Indeed, in that case dissipation is known to play a role in the overall dynamics, e.g., in the changes of potential vorticity once gravity waves start to break [33]. Finally, it is interesting that $N / f$ scaling has also been advocated, for example, in the context of statistical mechanics of nondissipative geophysical flows [34].

\section{B. Nonlinear effects}

Small nonlinearities and negligible dissipation are just the beginning of the story, with these assumptions broken when overturning takes place. For example, it is known that in three-dimensional turbulence without waves, the rate of energy dissipation can be evaluated phenomenologically as $\epsilon \sim U_{0}^{3} / L_{0}$, no matter how high the Reynolds number; this has been demonstrated using highly resolved direct numerical simulations [35] up to grids of $4096^{3}$ points [for the case of a coupling to a magnetic field, in which case Alfvén waves are present and interact with the flow, see [36] in two dimensions (2D), and [37] in three dimensions (3D)].

Similarly, there is a vast literature which concerns itself with the weak nonlinear coupling of waves, e.g., through resonant interactions [38], through weak turbulence theory (see [39] for the rotating case, and [40] for the stratified case), through turbulence closures [30,41,42], and more recently through asymptotic approaches [43-45]. In all cases, when the rotation or stratification is not strong enough and/or when the Reynolds number is high enough (a situation described by both the buoyancy Reynolds number $\mathcal{R}_{B}$, and what can be called the inertial Reynolds number $\mathcal{R}_{I}$, defined as

$$
\mathcal{R}_{B}=\operatorname{ReFr}^{2}, \quad \mathcal{R}_{I}=\operatorname{ReRo}^{2},
$$

being large enough), nonlinear couplings will take place between eddies and waves, sufficiently so that the energy will be transferred to small scales in a self-similar manner. Let us note here that in the following (see also Table I), $\mathcal{R}_{B, I}$ are evaluated at the peak of enstrophy, using dynamical variables, i.e., based on the so-called integral scale $L_{\text {int }}=\int\left[E_{V}(k) / k\right] / E_{V}$.

In these cases, that we will broadly call wave turbulence, the scaling laws for either rotating or stratified flows have been deduced both phenomenologically and analytically in the framework of the aforementioned closures and theories, and a continuous power-law spectrum is expected, steeper than a Kolmogorov spectrum, because of the weakening of interactions in the presence of waves. The energy flux $\epsilon$ is also 

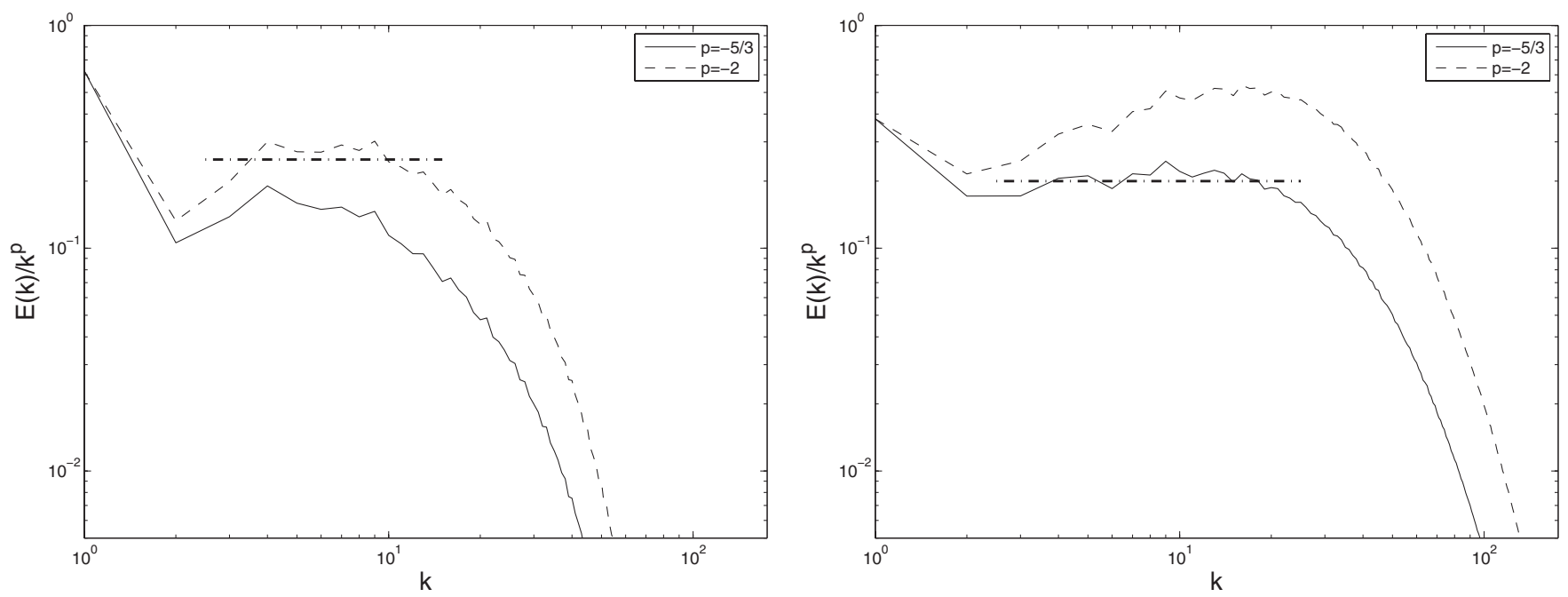

FIG. 1. $E(k) / k^{p}$, where $E(k)$ is the kinetic energy spectrum, averaged for one eddy turn-over time after the peak of enstrophy, and where $p$ is chosen to compensate for either a wave turbulence law $(p=-2$, dashed line) or a Kolmogorov law ( $p=-5 / 3$, solid line); $k$ is the isotropic wave number. Runs were computed on grids of $512^{3}$ points with initial conditions at $k_{0} \in[1,2]$, and Reynolds numbers $\operatorname{Re} \approx 10000$. The dash-dotted line indicates the best fit in the inertial range. Left: $N / f=2.99, \operatorname{ReRo}^{2} \approx 3.84, \operatorname{ReFr}=\operatorname{ReFr}{ }^{2} \approx 0.43$, with Fr $\approx 0.0063$ and Ro $\approx 0.019$. Right: $N / f=4.0, \operatorname{ReRo}^{2} \approx 1749, \operatorname{ReFr}^{2} \approx 109$, with $\mathrm{Fr} \approx 0.1$ and $\operatorname{Ro} \approx 0.4$. Note the steeper spectrum for moderate $N / f$ and

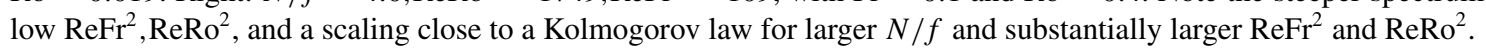

typically reduced, in general by a factor that is proportional to the ratio of the eddy-turn-over time to the time scale of the waves. Thus, we are in the presence of turbulence, but not the classical Kolmogorov turbulence, rather a wave turbulence regime that breaks down at small scales (beyond the Ozmidov scale). Two typical cases of the isotropic energy spectra are shown in Fig. 1. We observe well-developed kinetic energy spectra, rather steep for $N / f<3\left[E(k) \sim k^{-2}\right]$, whereas the power law is closer to a Kolmogorov law for $N / f=4$. In both cases, the dissipation scale $\eta$ (evaluated using a Kolmogorov spectrum) is barely resolved: One finds $\eta \approx 0.041$ and $k_{\eta} \approx$ 154 for the case where $N / f=2.99$, and $\eta \approx 0.044$ with $k_{\eta} \approx$ 144 for the case where $N / f=4.0$. Note that in the latter case, the turbulence is stronger and the spectra are not quite as well resolved, but the point of this study is not to examine Kolmogorov turbulence; in the presence of waves, one can simulate higher Reynolds numbers at a given resolution than in the absence of waves, again the set of governing parameters being $\operatorname{ReFr}^{2}$ and $\mathrm{ReRo}^{2}$ rather than $\mathrm{Re}$.

We do not intend to perform a detailed analysis of wavemode and vortical-mode perpendicular and parallel energy and helicity spectra here, but rather show that turbulence and helicity develop in the flows we study. There are several examples in the literature of such studies at high resolution for the energy [i.e., $E_{\perp, \|}\left(k_{\perp}, k_{\|}\right)$, see, for example, [46-48] for the purely stratified case, and [27] for rotating turbulence]. In our case, the choice of isotropic spectra is sufficient to show that there is indeed for these parameter regimes power-law spectra that develop through nonlinear mode coupling. These spectra may display intermittency at small scale, a phenomenon that would require substantially higher numerical resolutions to study.

As noted in Sec. III A, when the Reynolds numbers are increased, the amplitude of the nonlinear term can be expected to increase, and helicity (restricted to its evaluation using only $\mathbf{u}_{\perp}$ ) should be given by Eq. (8) written above, which is the general expression with only the assumption of stationarity and no dissipation. In the simulations presented in the following section, we compared the ratio of the second term to the first in Eq. (8). For simulations with $0.5 \leqslant N / f \leqslant 3$ and with $\operatorname{ReRo}^{2}<20$ and $\operatorname{ReFr}^{2}<20$, the amplitude of the second term is smaller than $10-20 \%$ of the first term in most of the runs, and increases for runs with larger values of $N / f$.

Therefore, we can expect that for very small values of the control parameters, and for flows for which the geostrophic and hydrostatic balance holds, the latter implying $\partial_{z} w=0$, the helicity should remain zero. As fluctuations develop, and as weak nonlinear perturbations come into play, small departures from geostrophy will develop allowing for nonzero correlations between buoyancy and vertical velocity, as appears in Eqs. (9) and (10), and as can also be expressed in two-scale turbulence closure formalisms. In that case, we can expect helicity to develop. Helicity should be created at large scales, where rotation and stratification dominate over the nonlinear term, and may be transferred to smaller scales. Finally, for runs with stronger nonlinearities and large values of the control parameters, we can expect deviations from the prediction in Eqs. (9) and (10), associated with the extra term in Eq. (8). A detailed study of these deviations is left for future work.

\section{PARAMETRIC STUDY}

We have performed nine runs on grids of $512^{3}$ points, and 36 runs on $256^{3}$ grids, up to the peak of dissipation and beyond, with similar (but not identical) random initial conditions and $N / f \in[1 / 2,16.7]$.

Figure 2 gives the temporal evolution of helicity (top and middle) for several runs at either fixed Fr or fixed Ro, and of kinetic enstrophy $Z_{V}$ (bottom) for several flows at fixed Fr; the potential enstrophy $Z_{P}$ shows a behavior similar to 


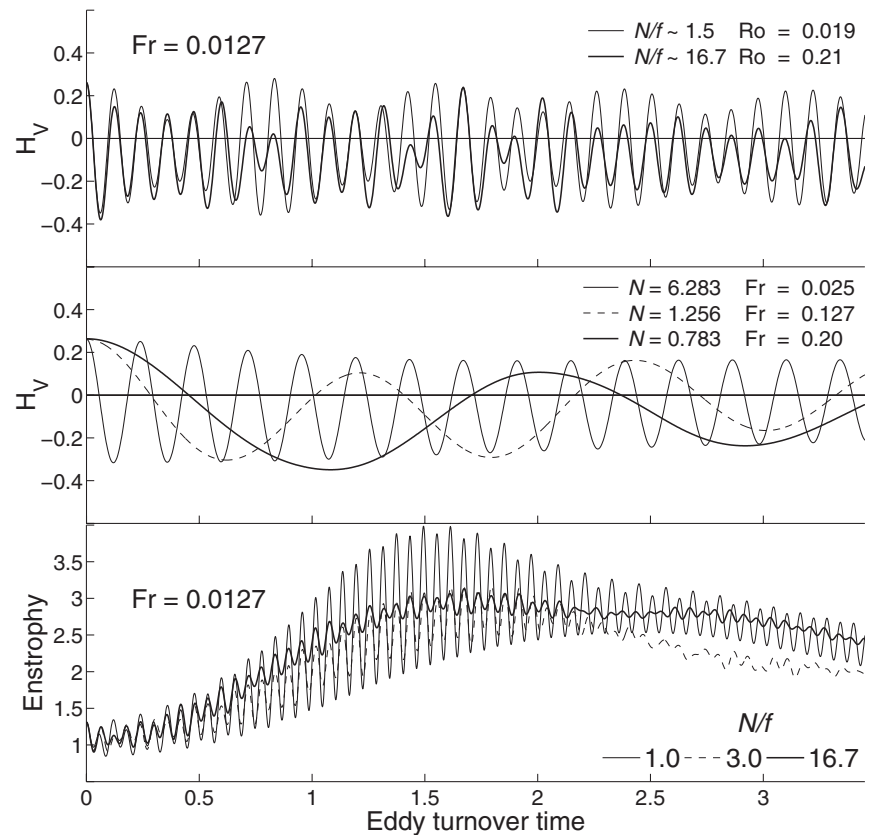

FIG. 2. Top and middle: Temporal evolution of the total helicity $H_{V}$ in several runs with different values of Fr, Ro, and $N$, as given by the labels. Note that the time averaged value of $H_{V}$ is negative, indicating negative helicity prevails in these runs even when the initial value of the helicity is positive. On top are runs with the same $\mathrm{Fr}$ whereas in the middle, runs with $N / f=1$ but with different Fr are shown. Bottom: Time evolution of the kinetic enstrophy $Z_{V}$ in runs with $\mathrm{Fr} \approx 0.01$ and $N=12.56$, and with different values of Ro. In all panels, oscillations are due to gravity waves, with their period proportional to $N$.

$Z_{V}$, with slightly smaller values. Note that in all quantities the oscillations are due to gravity waves because of the fact that our initial conditions are chosen to be unbalanced, and their periods are proportional to $N$. Across all runs, the maximum of $Z_{V}$ varies from 30 (for weak waves) to $\approx 2.5$, corresponding to the smallest Froude number considered. The time to reach this maximum varies from 1.5 to $3.2 \tau_{N L}$. The growth of enstrophy
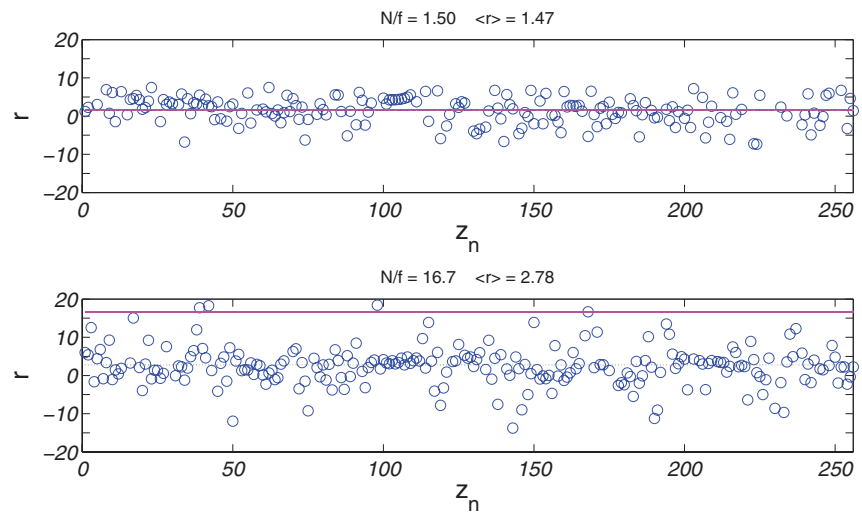

FIG. 4. (Color online) Variations of $r=-\left\langle H_{\perp}\right\rangle_{\perp, t} /\left\langle w \partial_{z} \theta\right\rangle_{\perp, t}$ [see Eq. (9)] with vertical layers of index $z_{n} ; n \in[1,256]$ is the index of the vertical plane, and the data are temporally averaged around the peak of enstrophy. The horizontal lines give the geostrophic balance prediction. Both runs are performed on grids of $256^{3}$ points with $\mathrm{Re} \approx 4 \times 10^{3}, \mathrm{Fr}=0.0127$, and $\mathrm{ReFr}^{2} \approx 0.672$. Top: $N / f=1.5, \operatorname{ReRo}^{2} \approx 1.51$. Bottom: $N / f=16.7, \operatorname{ReRo}^{2} \approx 186.6$. In the latter case, the prediction stemming from assuming weak nonlinearities no longer applies.

is typical of a turbulent flow, and is due to vortex stretching. The growth in the presence of waves is weaker, a characteristic of a wave turbulence regime.

The overall structures in this type of flows are shown in Fig. 3, which displays the volume rendering of buoyancy right after the peak of enstrophy for a run with $\mathrm{Fr}=0.1$ and $N / f=4$ (left), and for a run with $\mathrm{Fr}=0.025$ and $N / f=2$ (right), both performed on grids of $512^{3}$ points and with identical initial Reynolds numbers. The 3D rendering puts in evidence the stratification and the presence of large-scale layers; small-scale features with curved ribbons also occur for the run with smaller stratification. The run with $\mathrm{Fr}=0.1$ shows strong turbulent fluctuations, whereas the run with $\mathrm{Fr}=0.025$ is smoother, with weaker small-scale fluctuations.

We now examine the relation given by Eq. (9). In Fig. 4 is given the variation with the vertical index $z_{n}$ (i.e., the
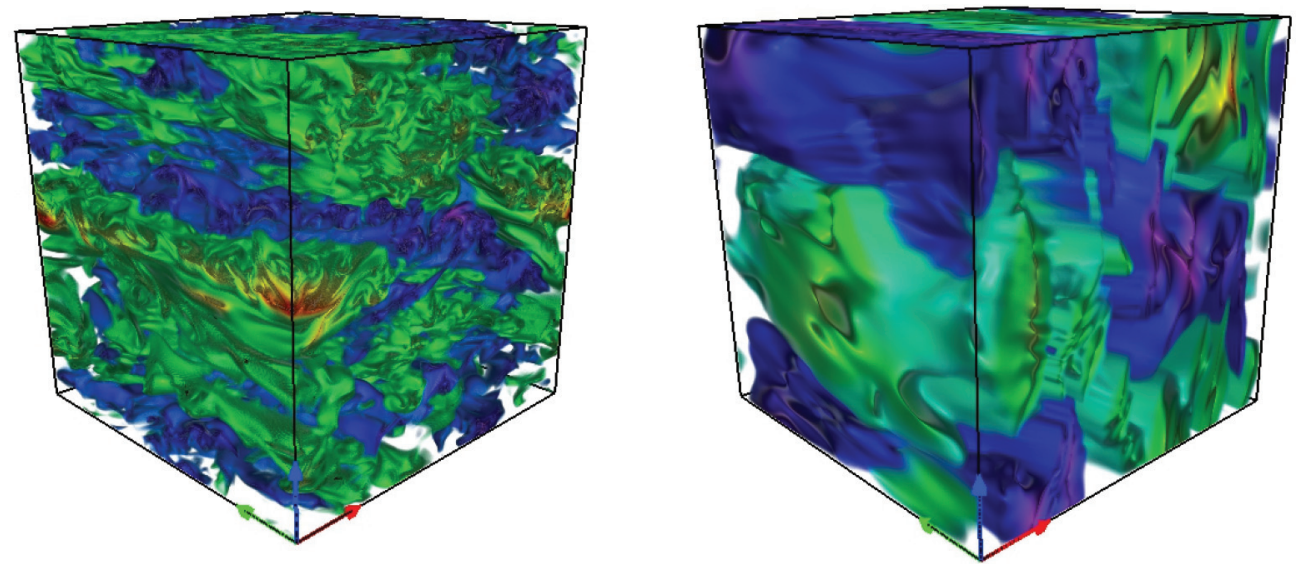

FIG. 3. (Color online) Visualization of the buoyancy $\theta$ in runs with $512^{3}$ grids, for $\operatorname{Re} \approx 10000, \mathrm{Fr}=0.1$, and $\mathrm{Ro}=0.4$ (left) and for the same $\operatorname{Re}, \mathrm{Fr}=0.025$, and $\mathrm{Ro}=0.05$ (right). The vertical direction is indicated by the blue arrow; dark (blue) and light (green) strata represent respectively positive and negative variations in $\theta$ around its mean, with sizable fluctuations and structuring, and with more turbulent eddies at higher Froude number. 
vertical grid point, in runs with $n_{p}=256$ ) of the ratio $r=-\left\langle H_{\perp}\right\rangle_{\perp, t} /\left\langle w \partial_{z} \theta\right\rangle_{\perp, t}$. The subindex $t$ indicates the quantities were not only averaged in planes perpendicular to $z$, but also averaged in time over the peak of enstrophy. The ratio is shown for two runs with different values of $N / f$, namely 1.5 (top) and 16.7 (bottom); the horizontal line gives the prediction based on weak nonlinearities, i.e., $N / f$. One observes regular variations around the mean value in the vertical, so in the following we shall perform vertical averaging as well. These fluctuations are likely associated with alternating quiescent and turbulent patches where the advection term is strong. In spite of these fluctuations, the run with $N / f=1.5$ shows good agreement with the prediction based on weak nonlinearities, while the run with $N / f=16.7$ does not. After performing a detailed analysis of all the runs, it is found that for all runs a good agreement with Eq. (9) is obtained for $N / f<3$; it is also fulfilled when $\mathrm{ReRo}^{2}<20$ together with $\mathrm{ReFr}^{2}<20$, i.e., for strong enough waves and weak nonlinearities as explained in the previous section. Note that the importance of the buoyancy Reynolds number has been identified previously, e.g., in the context of an emphasis on the role of anisotropy and the onset of Kelvin-Helmholtz instabilities due to vertical shearing [49].

Given the measurable vertical variations observed in Fig. 4, and following the expression in Eq. (9), we display in Fig. 5 (top) a scatter plot of $\left\langle H_{\perp}(t=0)\right\rangle_{\perp, z}-\left\langle H_{\perp}(t)\right\rangle_{\xi}$ as a function of $N / f\left\langle w \partial_{z} \theta\right\rangle$ for all runs with $N / f<3 ; \xi=\perp, t, z$ represents averaging on horizontal planes, for half an eddyturn-over time after the maximum of enstrophy, and over all the vertical planes as well. This allows for smoothing over temporal variations due to gravity waves, and over the vertical inhomogeneities of the flow that are inherent in strongly stratified flows as discussed before. The symbols in Fig. 5 indicate different Froude numbers, and the filled symbols are used for runs on grids of $512^{3}$ points. For the runs with $N / f<3$, all points lie close to a straight line with slope one, showing that the helicity created and the source of helicity according to Eq. (9) are linearly correlated. The result presented in the figure is robust for different choices of range over which the temporal average is performed, with windows of $0.5,1$ and 1.5 eddy-turn-over times after the peak of the enstrophy. When averaging over later times, say from 3.6 to 7.2 , good agreement with the prediction of helical geostrophic balance also holds, in part due to the fact that at late times, the Reynolds number has decreased and waves are now more easily predominant, with smaller Froude and Rossby numbers. It is interesting that the range of validity in $N / f$ corresponds in part to the range identified in [50] on the basis of a lack of resonant interactions for these parameters.

We also show in the shaded insets of Fig. 5 (top, middle) the same quantity for all the runs (i.e., all values of $N / f$, corresponding to all 9 runs with $512^{3}$ points and 36 runs with $256^{3}$ points). For $N / f>3$, the creation of helicity still occurs, though not quite at the level predicted by Eq. (9). The middle graph in Fig. 5 gives the same scatter plot but thresholded for the buoyancy and the inertial Reynolds numbers, $\operatorname{ReFr}^{2}, \operatorname{ReRo}^{2}<20$ (the inset shows again all points for comparison). In both cases of thresholding, about half the points are selected (roughly, 20), and the points in common between the top and middle scatter plots are $80 \%$ (namely,
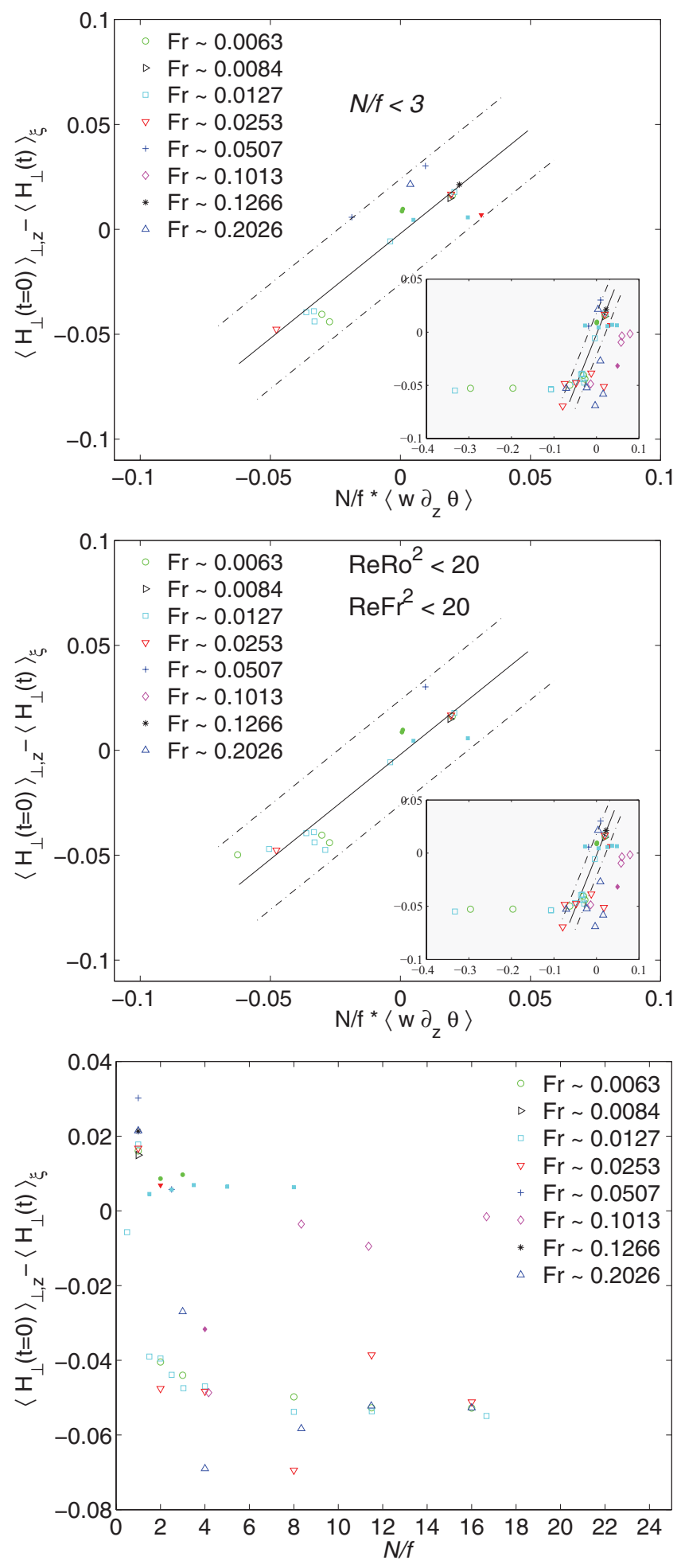

FIG. 5. (Color online) Helical geostrophic balance $\left\langle H_{\perp}(t=\right.$ $0)\rangle_{\perp, z}-\left\langle H_{\perp}(t)\right\rangle_{\xi}$ as predicted by Eq. (9), temporally and vertically averaged for runs with $N / f<3$ (top). In the shaded insets, the same data are given for all 45 runs of this study. Each symbol corresponds to a value of Fr (see labels), and the nine solid symbols indicate runs on grids of $512^{3}$ points at higher Reynolds numbers. In the middle graph, the points are selected using $\mathrm{ReFr}^{2}<20, \mathrm{ReRo}^{2}<20$ (the inset shows again all points for comparison). For completion the same scatter plot as a function of $N / f$ only, using the same symbols, is given at the bottom. 
16, see Table I). We can interpret this fact by saying that scales (temporal and spatial) are not sufficiently separated and it is difficult to sort out what may be the dominant effect for flows to obey the relationship of Eq. (9): a comparable rotation and stratification, or simply a low buoyancy Reynolds number. Finally, for completion, we give in Fig. 5 (bottom) $\left\langle H_{\perp}(t=0)\right\rangle_{\perp}-\left\langle H_{\perp}(t)\right\rangle_{\xi}$ as a function of $N / f$ alone. Note how most of the points pile up near negative values, even for large values of $N / f$. Since a growth of net helicity, and similarly of relative helicity, is not observed in freely decaying 3D homogeneous turbulence, with no rotation and no stratification, this further confirms that the production of helicity is characteristic of the regime under study. We finally note that it seems to be controlled more by the imposed stratification than by the Rossby number, in agreement with the fact that in rotating turbulence, helicity is conserved in the absence of dissipation.

\section{CONCLUDING REMARKS}

A parametric study of decaying rotating stratified turbulence shows that helicity is spontaneously produced at large scales, and that for $N / f<3$ (or, $\operatorname{ReFr}^{2}<20$ together with $\operatorname{ReRo}^{2}<20$ ), its value is associated with correlations between buoyancy and vertical shear, as derived in [21] (for nonstratified flows see [44], and for the magnetic case see [51]). This creation of helicity still takes place for larger values of $N / f$, and thus confirms the possibility, for geophysical and astrophysical flows, that the combination of rotation and stratification creates helicity which in turn can be the source of large-scale magnetic fields, as observed in stars and planets. Helicity production in rotating stratified flows can also be related to the observation of large-scale helicity in the atmosphere of the Earth, although it is not occurring in our study through an instability involving anisotropic small-scale helicity as studied before in $[52,53]$, but rather through a quasilinearization of the large-scale dynamics. Such large-scale helical flows might be relevant to the persistence of large-scale convective storms and to the onset phase of hurricanes $[4,53]$. It has also been shown that helical motions can be associated with the spiral rainbands of hurricanes when taking moisture into account in the dynamical equations [31]. The observed saturation in the level of helicity for larger values of $N / f$ and for sufficiently strong stratification can likely be understood in terms of the presence of vertically sheared horizontal flows in that regime (see, e.g., $[43,50]$ ), a tendency that persists in the absence of rotation [54]. The generation of helicity requires an interplay between stratification and rotation, and when stratification dominates, vertical and horizontal motions are less correlated. For large values of $\mathrm{ReFr}^{2}$ and $\mathrm{ReRo}^{2}$, the deviations from the prediction assuming weak nonlinearities can also be associated with an increase of the amplitude of the nonlinear term in the momentum equation, as verified in the simulations by direct estimation of the amplitude of the different terms in the equation for the helicity. The fact that rotating stratified flows can spontaneously produce large-scale helicity opens new lines of research and questions. For example, is there a detailed role to be played by potential vorticity conservation on the emergence of helicity? How would the inclusion of either shear, radiation, moisture, or some general forcing in Eq. (3) modify these results? And finally, how would turbulence affect significantly the creation of helicity, as the Reynolds numbers are further increased? Indeed, mixing is thought to have two transitions in terms of $\mathrm{ReFr}^{2}$ : In the presence of an imposed shear, it was shown in [55] (see also [16]) that below 7 , molecular diffusion is observed, with basically no turbulence; the intermediate regime $7<\operatorname{ReFr}^{2}<100$ follows a linear Osborn diffusion law [56], and above that value, a new regime is reached with diffusivity scaling as $\left(\operatorname{ReFr}^{2}\right)^{1 / 2}$. The latter regime is of course what matters for geophysical flows with $\mathrm{ReFr}^{2} \approx 10^{8}$, such as in the meridional overturning circulation, central to climate dynamics. We thus plan to pursue this study concerning the role of helicity in rotating stratified turbulence to help decipher the different mechanisms at play, a study that will eventually lead to better subgrid scale models of such flows that are needed to obtain a more accurate representation of enhanced diffusivities in weather and climate models.

\section{ACKNOWLEDGMENTS}

We thank an anonymous referee for remarks that led to clarifications and improvements of the paper. This work was sponsored by an NSF/CMG Grant No. 1025183 and by an NSF cooperative agreement through the University Corporation for Atmospheric Research on behalf of the National Center for Atmospheric Research (NCAR). Computer time was provided by NSF under the sponsorship of NCAR. For useful discussions we also acknowledge Alain Pumir, Alain Noullez, and Cecilia Rorai.
[1] P. W. Anderson, Science 177, 393 (1972); G. Falkovich, J. Phys. A: Math. Theor. 42, 123001 (2009).

[2] Y. Forterre and J. Dumais, Science 333, 1715 (2010); Y. Sawa, F. Yeb, K. Urayama, T. Takigawa, V. Gimenez-Pinto, R. L. B. Selinger, and J. V. Selinger, Proc. Natl. Acad. Sci. USA 108, 6364 (2011).

[3] P. MacCready and W. Rockwell Geyer, Ann. Rev. Marine Sci. 2, 35 (2010).

[4] E. Rassmussen and D. Blanchard, Weath. For. 13, 1148 (1998).

[5] H. K. Moffatt and A. Tsinober, Ann. Rev. Fluid Mech. 24, 281 (1992).
[6] M. E. McIntyre, Adv. Geo. Sci. 15, 47 (2008).

[7] W. H. Matthaeus, A. Pouquet, P. D. Mininni, P. Dmitruk, and B. Breech, Phys. Rev. Lett. 100, 085003 (2008).

[8] R. H. Kraichnan and D. Montgomery, Rep. Prog. Phys. 43, 547 (1980).

[9] C. Cichowlas, P. Bonaït, F. Debbasch, and M. Brachet, Phys. Rev. Lett. 95, 264502 (2005).

[10] A. J. Majda and X. Wang, Nonlinear Dynamics and Statistical Theories for Basic Geophysical Flow (Cambridge University Press, Cambridge, England, 2006); L. Bourouiba, Phys. Fluids 20, 075112 (2008). 
[11] J. B. Taylor, Phys. Rev. Lett. 33, 1139 (1974); P. Démoulin, Ann. Geophys. 25, 1 (2008).

[12] A. Brandenburg and K. Subramanian, Phys. Rep. 417, 1 (2005); M. Miesch and J. Toomre, Ann. Rev. Fluid Mech. 41, 317 (2009).

[13] C. Garrett and W. Munk, Ann. Rev. Fluid Mech. 11, 33 (1979).

[14] K. L. Polzin and Y. V. Lvov, Rev. Geophys. 49, 2010 RG000329 (2011).

[15] J. Gula and V. Zeitlin, J. Fluid Mech. 659, 69 (2010).

[16] G. Ivey, K. Winters and J. Koseff, Ann. Rev. Fluid Mech. 40, 169 (2008); E. Lindborg and G. Brethouwer, J. Fluid Mech. 614, 303 (2008).

[17] I. Fer, Deep Sea Res. II 53, 77 (2006); R. Ibragimov and V. Vatchev, Phys. Lett. A 376, 94 (2011).

[18] P. D. Mininni and A. Pouquet, Phys. Fluids 22, 035105 (2010); 22, 035106 (2010).

[19] P. Bartello, J. Atmos. Sci. 52, 4410 (1995); J. J. Riley and M.-P. Lelong, Ann. Rev. Fluid Mech. 32, 613 (2000).

[20] O. Praud, J. Sommeria, and A. Fincham, J. Fluid Mech. 547, 389 (2006); E. Lindborg, ibid. 550, 207 (2006); B. Galperin, S. Sukoriansky, and N. Dikovskaya, Phys. Scr., T 132, 014034 (2008).

[21] R. Hide, Geophys. Astrophys. Fluid Dyn. 7, 157 (1976); see also H. K. Moffatt, Magnetic Field in Electrically Conducting Fluids (Cambridge University Press, Cambridge, England, 1983).

[22] D. Lilly, J. Atmos. Sci. 43, 126 (1986).

[23] J. Molinari and D. Vollaro, J. Atmos. Sci. 67, 274 (2010).

[24] A. Sen, P. D. Mininni, D. Rosenberg, and A. Pouquet, Phys. Rev. E 86, 036319 (2012); A. Pouquet, A. Sen, D. Rosenberg, P. D. Mininni, and J. Baerenzung, Physica Scripta (in press).

[25] C. Nappo, in An Introduction to Atmospheric Gravity Waves (Academic, New York, 2002), p. 276.

[26] H. Aluie and S. Kurien, Europhys. Lett. 96, 44006 (2011).

[27] P. D. Mininni, D. Rosenberg, and A. Pouquet, J. Fluid Mech. 699, 263 (2012)

[28] P. D. Mininni, D. Rosenberg, R. Reddy, and A. Pouquet, Parallel Computing 37, 316 (2011).

[29] J. Clyne, P. D. Mininni, A. Norton, and M. Rast, New J. Phys. 9, 301 (2007).

[30] L. Bourouiba and P. Bartello, J. Fluid Mech. 587, 139 (2007).

[31] Y. Xu and R. Wu, Adv. Atm. Phys. 20, 940 (2003).
[32] C. Rorai, D. Rosenberg, A. Pouquet, and P. D. Mininni, arXiv:1211.3166.

[33] P. Haynes and M. McIntyre, J. Atmos. Sci. 42, 2021 (1990).

[34] G. Holloway, Ann. Rev. Fluid Mech. 18, 91 (1986).

[35] T. Ishihara, T. Gotoh, and Y. Kaneda, Ann. Rev. Fluid Mech. 41, 165 (2009).

[36] D. Biskamp and H. Welter, Phys. Fluids B 1, 1964 (1989); H. Politano, A. Pouquet, and P. L. Sulem, ibid. 1, 2330 (1989).

[37] P. D. Mininni and A. Pouquet, Phys. Rev. E 80, 025401(R) (2009).

[38] K. Hasselman, J. Fluid Mech. 12, 481 (1962); F. Bretherton, ibid. 20, 457 (1964).

[39] S. Galtier, Phys. Rev. E 68, 015301(R) (2003).

[40] Ph. Caillol and V. Zeitlin, Dyn. Atmos. Oceans 32, 81 (2000).

[41] L. Jacquin, O. Leuchter, C. Cambon, and J. Mathieu, J. Fluid Mech. 220, 1 (1990); C. Cambon, R. Rubinstein, and F. S. Godeferd, New J. Phys. 6, 73 (2004).

[42] F. Bellet, F. S. Godeferd, J. F. Scott, and C. Cambon, J. Fluid Mech. 562, 83 (2006).

[43] P. F. Embid and A. J. Majda, Geophys. Astrophys. Fluid Dyn. 87, 1 (1998).

[44] R. Klein, Ann. Rev. Fluid Mech. 42, 249 (2010).

[45] K. Julien, E. Knobloch, R. Milliff, and J. Werne, J. Fluid Mech. 555, 233 (2006).

[46] D. Fritts, L. Wang, J. Werne, T. Lund, and K. Wan, J. Atmos. Sci. 66, 1126 (2009).

[47] Y. Kimura and J. Herring, J. Fluid Mech. 698, 19 (2011).

[48] S. Almalkie and S. M. de Bruyn Kops, J. Turb. 13, N29 (2012).

[49] J. J. Riley and S. M. de Bruyn Kops, Phys. Fluids 15, 2047 (2003); A. Deloncle, P. Billant, and J.-M. Chomaz, J. Fluid Mech. 599, 229 (2008).

[50] L. Smith and F. Waleffe, J. Fluid Mech. 451, 145 (2002).

[51] H. K. Moffatt, in IUTAM Book Series, edited by Y. Kaneda, Vol. 4 (Springer-Verlag, Berlin, 2008), p. 331.

[52] U. Frisch, Z. She, and P. Sulem, Physica D 28, 382 (1987).

[53] G. Levina and M. Montgomery, Dokl. Earth Sc. 434, 1285 (2010).

[54] F. Godeferd and C. Cambon, Phys. Fluid 6, 2084 (1994).

[55] L. Shih, J. Koseff, G. Ivey, and J. Ferziger, J. Fluid Mech. 525, 193 (2005).

[56] T. R. Osborn, J. Phys. Oceanogr. 10, 83 (1980). 